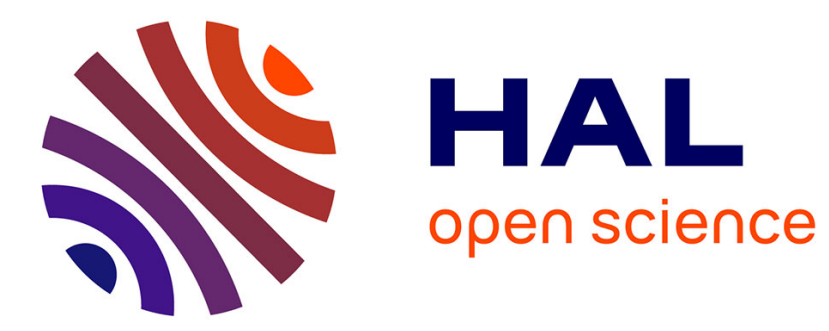

\title{
Radar Optimal Times Detection Allocation in Multitarget Environment
}

Marie de Vilmorin, Emmanuel Duflos, Philippe Vanheeghe

\section{To cite this version:}

Marie de Vilmorin, Emmanuel Duflos, Philippe Vanheeghe. Radar Optimal Times Detection Allocation in Multitarget Environment. IEEE Systems Journal, 2009, Systems Journal, IEEE, 3 (2), pp.210-220. 10.1109/JSYST.2009.2017393 . inria-00448956

\section{HAL Id: inria-00448956 https://hal.inria.fr/inria-00448956}

Submitted on 20 Jan 2010

HAL is a multi-disciplinary open access archive for the deposit and dissemination of scientific research documents, whether they are published or not. The documents may come from teaching and research institutions in France or abroad, or from public or private research centers.
L'archive ouverte pluridisciplinaire HAL, est destinée au dépôt et à la diffusion de documents scientifiques de niveau recherche, publiés ou non, émanant des établissements d'enseignement et de recherche français ou étrangers, des laboratoires publics ou privés. 


\title{
Radar Optimal Times Detection Allocation in Multitarget Environment
}

\author{
Marie Godin de Vilmorin, Emmanuel Duflos Senior Member, IEEE , Philippe \\ Vanheeghe Senior Member, IEEE
}

\begin{abstract}
This paper deals with the problem of the management of Electronically Steered Antenna (ESA) in multitarget environments. Radars are used to detect, locate and identify targets. In this paper we focus on the detection of several aerial targets in a fixed given time. The difficulty of such detection lies in the fact that targets may be located anywhere in the space, but radars can only observe a limited part of it at a time. As a result, it is necessary to change their axis position over time. This paper describes the main steps to derive an optimal radar management in this context: the modeling of the radar, the determination of a criterion based on the target detection probability and the temporal optimization process leading to sensor management strategy. An optimization solution is presented for several contexts and several hypotheses about prior knowledge concerning the targets' locations. First, we propose a method for the optimization of the radar detection probability in a single target environment. It consists in the decomposition of the detection step into an optimal number of independent elementary detections. Then, in a multitarget context with deterministic prior knowledge, we present an optimal time allocation method which is based on the results of non linear programming. Finally, in a multitarget context with probabilistic prior knowledge, results in Search Theory are used to determine an optimal temporal allocation.
\end{abstract}

\section{Index Terms}

Sensor Management, Radar Management, Detection, Optimal Temporal Allocation

\section{INTRODUCTION}

Sensors are more and more utilized as part of multisensor systems. In such systems, each sensor brings its complementarity and its redundancy. The complexity and performance of many sensors have also increased, leading to more complex multisensor systems supplying decision centers with increasing amounts of data.

In order to take the best advantage of multisensor systems, it is important not to consider each sensor individually as a passive measuring system. Instead, the interactions between the different sensors should be taken into account in order to use them in a globally optimal manner. Multisensor system management is however very complex. It is often impossible for a person to find an optimal solution, so that automated multisensor management strategies must be conceived.

From a theoretical point of view this problem can be written in the optimization framework, and the sensor management viewed as a Markov decision problem. Unfortunately, the complexity is such that it is impossible in practice to derive or implement optimal solutions. Alternative approaches leading to sub-optimal solutions have thus been proposed. In [1], [2] or [3] the authors use reinforcement learning, Qlearning and approximation functions to derive sub-optimal solutions. In these and other works, the choice of the next action is based on information theory using information divergence metrics like the Rényi information divergence and the Kullback Leibler divergence [1], [2], [4], [5]. In [6] Mahler proposes to solve the problem in the random sets framework. A review of the mathematical tools for sensor

Received Date: November 15, 2007, Revised Date: November 18, 2008.

Marie Godin de Vilmorin is with the LGIA, 9 rue du Temple, BP 665, 62030 Arras Cedex, France

Emmanuel Duflos is with the LAGIS, UMR CNRS 8146, INRIA Lille - Nord Europe, SEQUEL team, Ecole Centrale de Lille, BP48, 59651 Villeneuve d'Ascq Cedex, France

Philippe Vanheeghe is with the LAGIS, UMR CNRS 8146, INRIA Lille - Nord Europe, SEQUEL team, Ecole Centrale de Lille, BP48, 59651 Villeneuve d'Ascq Cedex, France 
management can be found in [7]. These works provide possible solutions to the multisensor management problem. However, to the knowledge of the authors, it is generally difficult to derive performance bounds for them, which can be a drawback in an operational context. Moreover, these approaches rarely take into account the characteristics of the sensors.

In this paper, we consider the case of the supervision of an aerial space, where several aircrafts are targets, and other ones are equipped with ESA radars and are in charge of the observation of the targets. This aerial space supervision supports a more general systems that aims at detecting the targets, estimating their positions and identifying them.

The sensors are ESA radars, for which the orientation of the field of view can be achieved without any inertia ([8], [9]), and with which the steps of detection, estimation and identification can be carried out almost simultaneously.

[10] presents the classical methods for time splicing between these three steps. Here, we focus on the detection step for which a fixed known duration $T$ has been allocated by an underlying time splicing process. Methods, such as in [10] or [11], have already been developed for the optimization of the detection of a single target by a radar. Intead, we aim here at globally optimizing the detection of all the targets present in the aerial space. More exactly, the problem that we aim to solve is the following: if a radar has to detect $P$ targets during a known fixed duration $T$, how must it be managed so that it can detect each of them as well as possible? In other words, how does it optimally distribute the duration $T$ over the space directions to global optimize the probability of detection of all targets?

This article proposes a method for optimal temporal allocation based on the modeling of the sensor and assuming some prior knowledge about the targets' locations. The underlying hypotheses of this work are presented in Section II. We then propose in Section III a modeling of the radar detection functions. These functions are used to establish an optimal management strategy of the sensor in a monotarget environment. Some analytic results and a performance evaluation are also presented. Section IV then considers multiple targets. Under the assumption of a deterministic prior knowledge, we propose an optimal temporal allocation that uses the results of Section III. In Section V, the context is still multitarget but we assume a probabilistic knowledge about the targets' locations, instead of the deterministic knowledge considered in Section IV. An optimal temporal allocation strategy is presented that is derived from the Search Theory ([12], [11], [13], [14], [15]). Finally, some illustrative simulations are presented in Section VI.

The results presented in this article are extracted from [16].

\section{HYPOTHESES}

The principal assumption of this article is the prior knowledge about the locations of the targets. This assumption is justified by the integration of the sensor in a supervision system of the ESM type (Electrical Support Measurement) or AEW type (Airbone Early Warning) which provide information on the range and angular positions of the targets. Such systems may however estimate range and angular location stochastically. In Sections III (monosensor monotarget context) and IV (monosensor multitarget context), we first consider deterministic estimations of the targets' locations. Then, in Section V (monosensor multitarget context), we consider the more realistic case of stochastic estimations.

Another assumption is the spatial context: we consider a 2D space. This assumption reduces calculations, but does not limit the general character of the study.

Finally, we suppose that the observation durations are sufficiently short so that the aircrafts can be considered stationary. This assumption allows us to consider that targets do not move out of resolution cells during an observation (the notion of resolution cell is presented in the Section III).

\section{DETECTION PROBABILITY OPTIMIZATION}

\section{A. The radar sensor}

In order to establish optimal multisensor management strategies, it is necessary to understand the operating mode of the sensor. The radar considered here is an ESA radar, which is an active sensor: it 

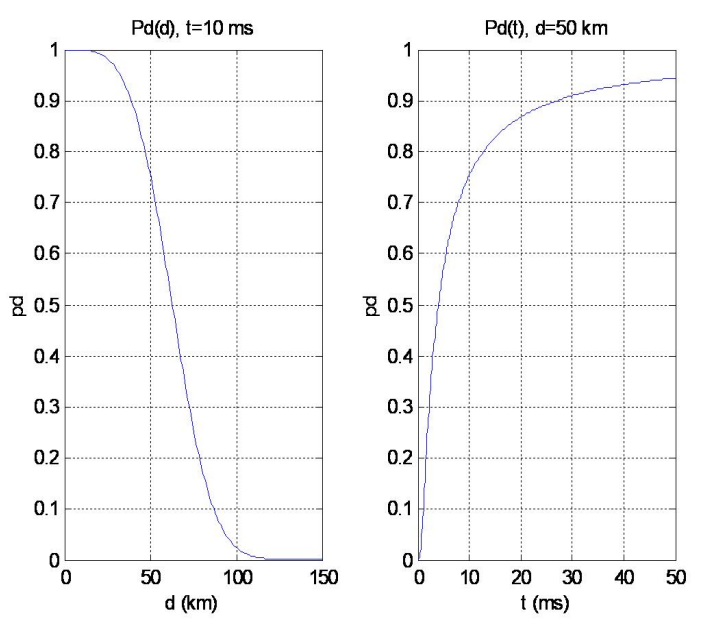

Fig. 1. Evolution of detection probability with respect to range and time $\left(P_{f a}=10^{-8}\right.$, false alarm probability)

emits a signal which is reflected on the target. Such a radar also presents the particularity that its mechanical axis is fixed, the analyzing beam direction being modified electronically during the observation.

The modeling of the detection functions depends on the characteristics of the target. However, a target does not have a regular form, so that the returned energy varies from an impulse to another. The target can then be considered as a set of elementary reflectors which positions in space are related to the target orientation. The returned signals are then independent and the amplitude of the received energy fluctuates. These targets are thus called "fluctuating targets". Different models of received energy have been proposed in [17], [18] and [19], and are called Swerling 0, Swerling 1, Swerling 2, Swerling 3 and Swerling 4. The Swerling 1 type, used in this work, is particularly adapted to the case of the air target detection.

If the sensor observes during a duration $T$ a target at the range $r$ in a direction which forms an angle $\theta$ with the mechanical axis of the antenna, then the signal to noise ratio of an echo is equal to ([19]):

$$
S N R=\frac{\alpha T \cos ^{2}(\theta)}{r^{4}}
$$

where $\alpha$ is an operational parameter that depends on the radar and some target features (target's radar cross section) [19]. Targets are supposed to have the same reflexion power, thus $\alpha$ is constant. Equation (1) is established with the disturbing signal, which is supposed to be only due to the thermal noise of the radar, modeled with a normal random variable. Calculation details and $\alpha$ calibration are given in appendix A.

We can now write the expression of the target detection probability:

$$
P_{d}=\left(P_{f a}\right)^{\frac{1}{1+S N R}}
$$

where $P_{f a}$ is the false alarm probability. Equation (2) is established under the assumptions of a fluctuating target and a modeling of the received energy of type Swerling 1 ([8]). Calculation details are given in appendix B. Figure 1 illustrates the evolution of this detection probability with respect to range for a given time and with respect to time for a given range.

The value of the false alarm probability corresponds here to one false alarm per second and per resolution cell. We mean by resolution cell one interval in angle and range. The angle interval is the field of view of the sensor, and the range interval corresponds to an impulse of duration $\tau$ :

$$
\tau=\frac{2 d_{p}}{c}
$$

where $c$ is the speed of light and $d_{p}$ is the length of the interval, i.e. the length of the cell. 


\section{B. Optimization of the detection probability in a monosensor monotarget context}

1) Problem statement: According to Equations (1) and (2) and Figure 1, the detection probability is strongly degraded when the range increases. Several methods exist to improve this. A first method is to use a procedure of "alert and confirmation" ([20], [10]). This method consists in doing two detection steps: the first one with a low detection threshold, the second one with a higher one in order to eliminate false alarm stemed from the alert step. During the second step, the form of the emitted wave is modified with respect to the target features. The problem is that this process of decomposition in two steps needs a long integration time. A solution could be to increase this decomposition time but this presents some disadvantages:

- for high detection probability, the slope $\frac{d P_{d}}{d t}$ is small,

- the use of long $T$ can be non appropriate because of the non stationarity of the target.

Another solution is then to change the mode of use of the sensor. Instead of acquiring only one signal during $T$ and performing the detection based on this single signal, $N$ elementary signals are acquired during $T$ with different emission frequencies and $\mathrm{N}$ elementary detections are respectively carried out ([21], [22]). Since the elementary signals have distinct emission frequencies, the respective elementary detections are independent ([19], [9]), and the cumulative detection probability can be calculated as ([8]):

$$
P_{d}=1-\left(1-P_{d e}\right)^{N}
$$

where $P_{d e}$ is the elementary detection probability given by (2) with an observation duration equal to $\frac{T}{N}$. The problem is then to find the number $N$ of elementary detections which optimizes the cumulative detection probability.

2) Determination of the optimal number of elementary detections: By considering a target's signal to noise ratio far higher than one, it is possible to write the analytical expression of the elementary detection probability as:

$$
P_{d e}=\left(P_{f a}\right)^{\frac{1}{S N R}}=\exp \left(\frac{r^{4} N \ln \left(P_{f a}\right)}{\alpha T \cos ^{2}(\theta)}\right)
$$

We define the constant $\beta=\frac{r^{4} \ln \left(\frac{1}{P_{f a}}\right)}{\alpha T \cos ^{2}(\theta)}$, so that this probability can be more simply written as $P_{d e}=$ $\exp (-\beta N)$, and the cumulative detection probability (Equation (5)) becomes:

$$
P_{d}=1-\exp (N \ln (1-\exp (-\beta N)))
$$

We differentiate this expression with respect to $N$ in order to determine its value, $N_{\text {opt }}$, for which the probability is maximum. Solving the equation $\frac{d P_{d}}{d N}=0$. is equivalent to find $\gamma_{r}$ so that $f\left(\gamma_{r}\right)=0$ with:

$$
\begin{gathered}
f\left(\gamma_{r}\right)=\left(1-\exp \left(-\gamma_{r}\right)\right) \\
\ln \left(1-\exp \left(-\gamma_{r}\right)\right) \\
+\gamma_{r} \exp \left(-\gamma_{r}\right)
\end{gathered}
$$

where $\gamma_{r}=-\beta N=-\ln \left(P_{d e}\right)$.

The equation $f\left(\gamma_{r}\right)=0$ has a single solution: $\gamma_{r}=\ln 2$. The optimal number of elementary detections is thus equal to:

$$
N_{o p t}=\frac{\gamma_{r} \alpha T(\cos (\theta))^{2}}{r^{4} \ln \left(\frac{1}{P_{f a}}\right)}
$$

When considering that the elementary detection probability is equal to 0.5 , the cumulative one is then equal to:

$$
P_{d}=1-\exp \left(-\frac{T}{\tau_{r}}\right)
$$




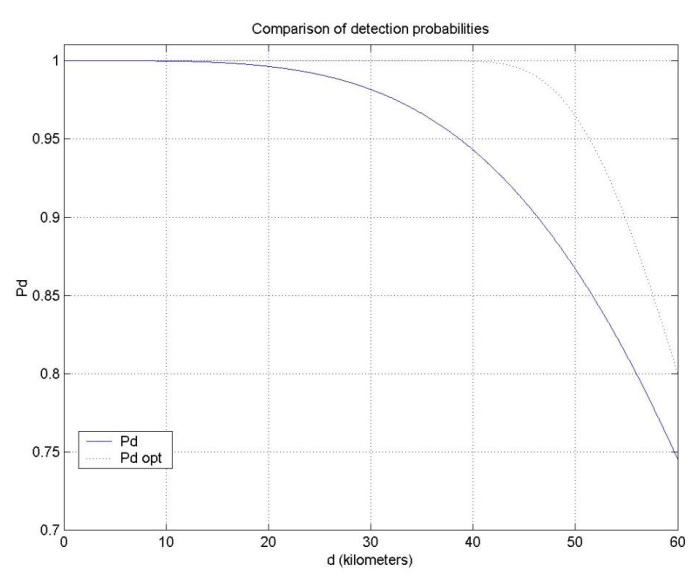

Fig. 2. comparison of detection probabilities, for a duration of $T=20 \mathrm{~ms}$

where

$$
\tau_{r}=\frac{r^{4} \ln \left(P_{f a}\right)}{\gamma_{r} \alpha(\cos (\theta))^{2} \ln \left(1-\exp \left(-\gamma_{r}\right)\right)}
$$

These results show that the modeling of the radar sensor detection functions makes possible the elaboration of analytical strategies for the optimization of the detection probability, and that it is possible to quantify the detection performance. These are determined under the hypotheses of a fluctuating target and a signal to noise ratio far higher than one.

Figure (2) illustrates these results by comparing the simulated performances of a more traditional strategy and the developed one using the decomposition of the detection. This simulation, obtained with a duration of $20 \mathrm{~ms}$, shows that when the target is distant of more than a few kilometers from the sensor, the performance of a strategy using the decomposition of the detection is better than the performance of the more traditional one.

A few remarks about these results:

- The optimal number $N_{\text {opt }} \notin \mathbb{N}$. However, this does not impact the generality of the method and allows to calculate optimal performance which may be used as reference, like the Cramer-Rao lower bound in estimation theory ([23]).

- The assumption of a large signal to noise ratio is a trick to write an analytical expression of the detection probability. However, the probabilities obtained can be close to 0.5, which justifies the elaboration of an optimal detection process.

In this Section, we have presented a method for optimizing the detection probability of a target by a radar sensor. Results are based on the modeling of the sensor. In the second Section, we will see how these results can be used for the detection of several targets.

\section{Monosensor multitarget CONTEXT With DETERMinistic KNOWLEDGE}

The study presented in the Section III-A deals with the single target detection optimization. But in reality the environment is generally multitarget. In this Section, we are thus interested in the global optimization of the detection of several targets. The assumptions about the prior knowledge described in Section II are still considered. Then, we assume that there are $P$ targets in the air space, for which the location is deterministically known. We also consider a given duration $T$ for their detection. Our aim is to optimize the use of this duration in order to optimize the global detection of the targets, knowing that the operating mode of the sensor allows it to change the axis of its fields of view instantaneously. We first define a criterion to optimize. 


\section{A. Criterion definition}

First, we ask ourselves what are the elements which must appear in the criterion. Functions expressing the detection performance must appear, but operational considerations must also be taken into account. It is more important to accurately detect the targets which represent a high threat than to detect all of them with a low degree of localization. Such a strategy leads to the introduction of ponderation taps.

We consider a situation in which the $P$ targets were already detected, and our goal is to detect them again, with another sensor for example. The knowledge about them is such that their angular deviations $\theta_{i}$ and their ranges $r_{i}$ are known, $\forall i \in\{1, . ., P\}$. Furthermore, targets are supposed to be located in different parts of the space, that is $\theta_{i} \neq \theta_{j} \forall(i, j) \in\{1, \ldots, P\}^{2}$. Let us call $t_{i}$ the duration of the observation of the target $i$ by the radar. Since angular deviations are different, the radar may not observe several targets simultaneously. This results into the following relation between the durations $t_{i}$ and the total duration $T$ :

$$
\sum_{i=1}^{P} t_{i}=T
$$

where:

$$
t_{i} \geq 0 \quad \forall i \in\{1, . ., P\}
$$

Durations are positive, but we will see that they could be null, mainly because of the relative positions of the targets with respect to the sensor.

Our aim is to maximize the detection of all the targets. As a probability is always positive, maximizing each of them is equivalent to maximizing their sum. We thus define the criterion:

$$
J=\sum_{i=1}^{P} \varepsilon_{i} P_{d i}\left(t_{i}\right)
$$

$\varepsilon_{i}$ is the ponderation tap for the target $i$ and can be interpreted as a threat or priority coefficient. This concept of threat is introduced in a general way, we are not really interested here in its characterization (coefficients can be inversely proportionnal to the distance, for example [24]); and $P_{d i}\left(t_{i}\right)$ is the probability of detection of the target $i$ by the radar sensor and for a duration $t_{i}$. If this duration is known, then we are in the monosensor monotarget context analyzed in Section III. Using previous results, that is a decomposition of the detection during $t_{i}$, it is possible to write $P_{d i}\left(t_{i}\right)$ like:

$$
P_{d i}\left(t_{i}\right)=1-\exp \left(-\frac{t_{i}}{\tau_{r_{i}}}\right)
$$

where:

$$
\tau_{r i}=\frac{r^{4} \ln \left(P_{f a}\right)}{\gamma_{r} \alpha\left(\cos \left(\theta_{i}\right)\right)^{2} \ln \left(1-\exp \left(-\gamma_{r}\right)\right)}
$$

According to Equation (14), $J$ will reach its maximum when the durations $t_{i}$ tend towards infinite, which is not compatible with the temporal constraint which was define. Our aim is thus to optimize the criterion $J$ under the equality constraint (13) and the $P$ inequality constraints (12):

$$
\mathcal{J}\left\{\begin{array}{l}
\text { maximize } \\
J=\sum_{i=1}^{P} \varepsilon_{i} P_{d i}\left(t_{i}\right) \\
\text { under the constraints } \\
\left\{\begin{array}{l}
\sum_{i=1}^{P} t_{i}=T \\
t_{i} \geq 0 \quad \forall i \in\{1, . ., P\}
\end{array}\right.
\end{array}\right.
$$




\section{B. Criterion optimization}

In the definition of the criterion we recognize a problem of optimization under constraints. First, the problem is formalized by the introduction of some notations. Let us define:

$$
\begin{gathered}
t=\left[t_{1}, t_{2}, \ldots, t_{P}\right]^{T} \\
f(t)=-\sum_{i=1}^{P} \varepsilon_{i} P_{d i}\left(t_{i}\right) \\
h(t)=\sum_{i=1}^{P} t_{i}-T \\
g_{i}(t)=t_{i}, \forall i \in\{1, . ., P\}
\end{gathered}
$$

$f$ is the objective function, $h$ and $g_{i}$ are the constraint functions. Considering the general form of the probabilities $P_{d i}$, this problem must be solved in the framework of non linear programming methods [25]. The problem is however simpler since the constraints are linear. We therefore reduce it to a linearly constrained optimization for which, as it is shown in Appendix C, the Karush-Kuhn-Tucker conditions can be applied. With the above notations, the maximization of the criterion (13) is equivalent to the minimization of the objective function $f$ under the same constraints. The objective function $f$ being defined on $\mathbb{R}^{P}$, the $P$ constraints $g_{i}(t) \geq 0$ lead us to search for a minimum on $\mathbb{R}^{P+}$. The optimization of the function $f$ can finally be written as a problem of convex minimization of a Lagrange function [25]:

$$
L(t, \lambda, \mu)=f(t)+\lambda h(t)+\sum_{i=1}^{P} \mu_{i} g_{i}(t)
$$

where $\lambda$ and $\mu=\left[\mu_{1}, \ldots, \mu_{P}\right]^{T}$ are the Lagrange multipliers.

Lemma 1: Let us introduce the function $x \mapsto\lfloor x\rfloor^{+}$defined on $\mathbb{R}$ by:

$$
\begin{aligned}
\lfloor x\rfloor^{+} & =x \text { if } x>0 \\
& =0 \text { else }
\end{aligned}
$$

and $\lambda$ the single solution of the following equation:

$$
\sum_{i=1}^{P} \frac{\tau_{r i}}{T}\left\lfloor\left.\ln \left(\frac{T \varepsilon_{i}}{\tau_{r i} \lambda}\right)\right|^{+}-1=0\right.
$$

If $\mathcal{I}$ is the suffix set defined by:

$$
\mathcal{I}=\left\{\forall i \in\{1, . ., P\} \mid \lambda<\frac{T \varepsilon_{i}}{\tau_{r i}}\right\}
$$

then the optimal temporal allocation which optimizes the Lagrangian (21) is given by these results:

$$
\left\{\begin{aligned}
t_{i} & =\tau_{r i} \ln \left(\frac{T \varepsilon_{i}}{\tau_{r i} \lambda}\right) \text { if } i \in \mathcal{I} \\
& =0 \text { else }
\end{aligned}\right.
$$

Furthermore, the optimal elementary detection number for each $t_{i}$, i.e. for each target, is equal to:

$$
n_{i}=\frac{\gamma_{r} \alpha t_{i}(\cos (\theta))^{2}}{r_{i}^{4} \ln \left(\frac{1}{P_{f a}}\right)}
$$

Proof: the demonstration of this lemma is given in appendix C. 
So far, we have derived an optimal allocation over a duration $T$. An implicit assumption has been made though: the infinite divisibility of the duration $T$, which is not true for all sensors, but is justified in the case of an ESA radar, for which the delay to move the beam from an angular position to another can be neglicted.

The set (25) depends on a parameter $\lambda$ which is the solution of the equation (68). This equation has an analytical solution if $\operatorname{card}(\mathcal{I})=P$.

Lemma 2: If $\operatorname{card}(\mathcal{I})=P$, then the optimal allocation of the duration $T$ between the $P$ targets is:

$$
t_{i}=\frac{\sum_{j=1}^{P} \tau_{r j} \ln \left(\frac{\epsilon_{i} \tau_{r j}}{\epsilon_{j} \tau_{r i}}\right)+T}{\sum_{j=1}^{P} \frac{\tau_{r j}}{\tau_{r i}}} \forall i \in\{1, \ldots, P\}
$$

A sufficient condition to obtain this result is that there exists $\lambda_{0}>\lambda$ such that:

$$
\lambda_{0}<\frac{T \epsilon_{i}}{\tau_{r i}} \forall i \in\{1, \ldots, P\}
$$

Proof: According to proof of the lemma 1, $\lambda$ is solution of the following equation:

$$
\sum_{i=1}^{P} \frac{1}{\omega_{i}} \ln \left(\frac{\epsilon_{i} \omega_{i}}{\lambda}\right)-1=0
$$

That is

$$
\underline{\lambda}=\exp \left(\frac{\left(\sum_{i=1}^{P} \frac{1}{\omega_{i}} \ln \left(\epsilon_{i} \omega_{i}\right)\right)-1}{\sum_{i=1}^{P} \frac{1}{\omega_{i}}}\right)
$$

By the introduction of this expression of $\lambda$ in (63), we obtain a new expression of $x_{i}$ :

$$
x_{i}=\frac{\left(\sum_{j=1}^{P} \frac{1}{\omega_{j}} \ln \left(\epsilon_{j} \omega_{j}\right)\right)+1}{\sum_{j=1}^{P} \frac{\omega_{i}}{\omega_{j}}}
$$

Finally, using Equation (56), we obtain the optimal temporal allocation (27). Moreover, if it exists $\lambda_{0}>\lambda$ such as the $P$ inequalities (28) are verified, then:

$$
\lambda<\frac{T \epsilon_{i}}{\tau_{r i}} \forall i \in\{1, \ldots, P\}
$$

and according to the definition of the set $\mathcal{I}$, we have $\operatorname{card}(\mathcal{I})=P$.

Lemmas 1 and 2 make the use of an optimal temporal allocation possible.

The major hypothesis of this section is the deterministic knowledge about the situation. Although it may be justified by the integration of the sensor in a supervision system, this assumption cannot seem very realistic in an operational context. The problem of optimal temporal allocation in the case of a prior knowledge defined by probability densities is thus investigated in Section V.

\section{Monosensor MUltitarget CONTEXT With PROBABILISTIC KNOWLEDGE}

We now suppose that the location of the target is uncertain, and modeled by probability density functions. In this context we have to consider the entire space and not only some specific directions. The optimal temporal allocation, that is the duration $t_{j}$ in a direction $j$ could be obtained using the previous results, provided the adaptation of the detection probability and the definition of a direction [16]. However, we introduce a theorical alternative: the Search Theory. It has appeared in the United States at the end of the World War II and deals with allocation of resources ([12], [11], [13]). More exactly, the fundamental problem is to allocate a given amount of search effort in order to maximize the probability of detecting an object located in a surveillance area. We mean by the term "search effort" all the potential resources used for the detection: material resources (e.g. sensors, fuel), unmaterial resources (e.g. observation time) or human resources. Many algorithms have been proposed over the last fifty years to solve this problem. We focus here on the "de Guenin" algorithm [14]. 


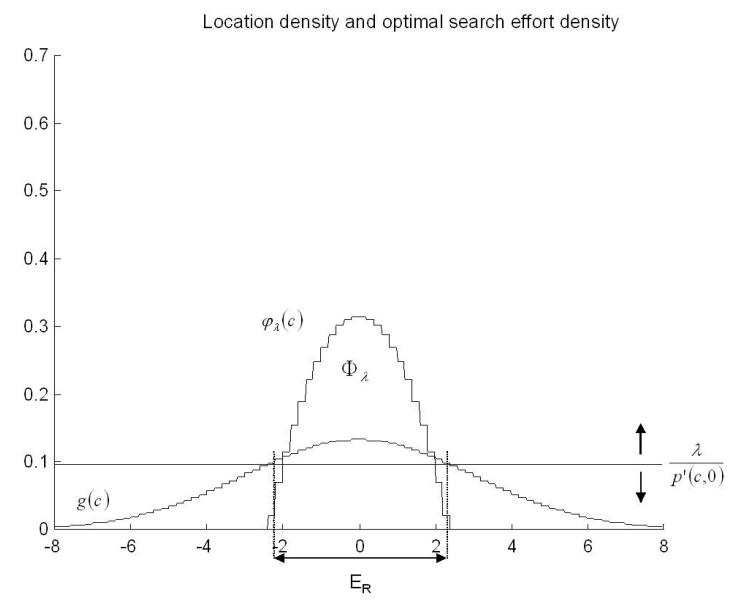

Fig. 3. Determination of the optimal search effort density, which satisfies $\sum_{c} \varphi(c)=\Phi$ from a location density

\section{A. The "de Guenin" algorithm}

Hypotheses are the following:

- one sensor must detect one target (monosensor monotarget environnment),

- the target is assumed stationnary,

- the sensor observation direction is fixed,

- the space is divided into cells,

- the total search effort is $\Phi$ and is infinitly divisible,

- the prior knowledge is a probability density.

The aim is to optimize the detection probability of the target. de Guenin has proposed in [14] an iterative algorithm which establishes an optimal allocation of the search effort $\Phi$ in the cells of a space $E$. He proved that a necessary condition for the detection probability to be maximum is that the search effort satisfies the following condition:

$$
g(c) p^{\prime}(c, \varphi)=\lambda=\text { constant }
$$

where $c$ is a cell, $g$ is the prior location probability in cell $c, p_{\varphi}^{\prime}$ is the derivative of the detection probability $p(c, \varphi)$ of a target with a search effort $\varphi$ when the target is indeed inside the cell $c$, and $\lambda$ is a constant. This condition is necessary and sufficient when the probability $p(c, \varphi)$ obeys the law of diminishing returns. In this case, it is possible to invert the expression (33) and to establish the expression of the search effort in a cell $c$ with respect to $\lambda$ :

$$
\varphi(c)=f\left(\frac{\lambda}{g(c)}\right)
$$

where $f$ is the reciprocal function of $p^{\prime}(c, \varphi)$.

From these properties, the aim of the "de Guenin" algorithm is to adjust the value of $\lambda$ in order to establish an optimal allocation of search effort and to satisfy the constraint $\sum_{c} \varphi(c)=\Phi$. Figure 3 gives an illustration of the optimal distribution of a search effort from a location probability, $E_{R}$ is the set of cells for which $g(c)>\frac{\lambda}{p^{\prime}(c, 0)}$, i.e. for which equation (34) is true. Indeed, by the law of diminishing returns, the derivative of the detection probability is decreasing, so $\frac{1}{p^{\prime}(c, \varphi)}>\frac{1}{p^{\prime}(c, 0)}$. According to equation (33), $g(c)=\frac{\lambda}{p^{\prime}(c, \varphi)}$, so $g(c)>\frac{\lambda}{p^{\prime}(c, 0)}$.

As we can see, the description of this problem is quite similar to our, and we are going to show how it can be adapted. 


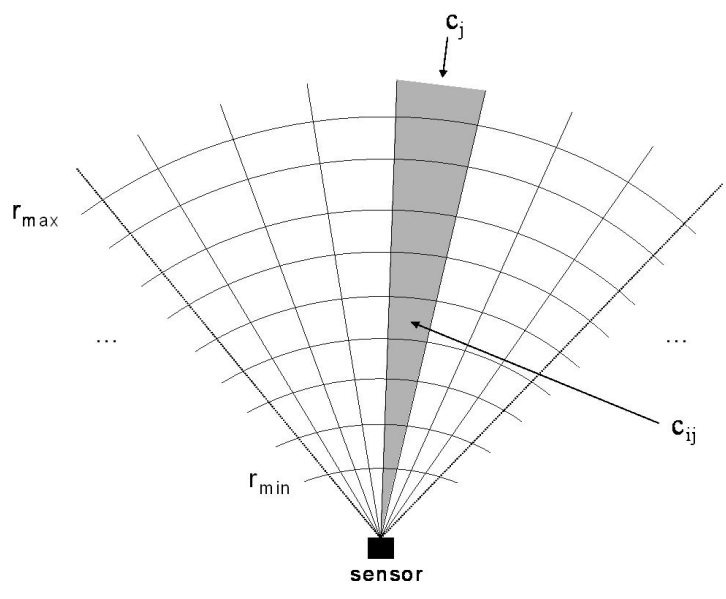

Fig. 4. Representation of the directions and cells of the space

\section{B. Application to our problem}

Let us make a comparison between the assumptions of the "de Guenin" algorithm and ours:

- the total search effort $\Phi$ corresponds to the total duration $T$. It is infinitly divisible,

- the set of cells corresponds to the space directions,

- $\varphi(c)$, the search effort in a cell $c$ corresponds to $t_{j}$, the observation duration in a direction $j$

These three assumptions are easily definable in our context. However, we consider a multitarget environment, whereas this algorithm is defined for a monotarget environment. Some adaptations are thus necessary:

- the prior knowledge corresponds to the probability density functions of localization. If the radar performs a new detection, then the probability densities are estimated based on the knowledge already acquired, else they are given by the supervision system in which the radar sensor is included.

- the location probability in a cell $c$ corresponds to the location probability of several targets in a same direction.

Let us see how these probabilities are calculated.

1) Probabilities calculation: Directions and cells are represented in figure 4.

The detection range interval is $\left[r_{\min }, r_{\max }\right] . c_{j}$ is an angular sector, its middle corresponds to the direction $j, j \in\left\{1, \ldots, N_{d}\right\}$. We have seen in the Section III-A that the sensor forms a set of range resolution cells in each direction, i.e. in each cell $c_{j}$. Then we consider a set of cells $c_{i j}$, at the range $r_{i}$, in the direction $j, i \in\left\{1, \ldots, N_{r}\right\}$. Since a sensor observes simultaneously all the targets present in the same direction, we are going to determine the probability of detecting from one to several targets in each direction. Thus, we will be in a configuration similar to the one of the "de Guenin" algorithm : a probability in each cell.

First we consider the expression of the detection probability given by Equation (5). It represents the detection probability of a target knowing that it is at a range $r$. Let $H_{k}$ be the event "the target $k$ is detected". By the formalism of conditionnal probabilities we have the probability of detecting the target $k$ at a range $r$ :

$$
P\left(H_{k}, r\right)=P\left(H_{k} \mid r\right) P(k, r)
$$

with $P(k, r)$ the $k^{\text {th }}$ target location probability at the range $r$.

Since the detection probability of a target in a given cell $c_{i j}$ can be approximated by the detection probability of this target assumed located at the center that cell [16], the expression of this probability can still be written:

$$
P\left(H_{k}, c_{i j}\right)=P\left(H_{k} \mid c_{i j}\right) P\left(k, c_{i j}\right)
$$


where $P\left(k, c_{i j}\right)$ is obtained by the integration of the density of probability in the cell $c_{i j}$. We note $P\left(k, c_{i j}\right)=\rho_{i j k} . P\left(H_{k} \mid c_{i j}\right)$ is calculated from expression (5), with the target at the approximate range $r_{i}$. Finally we obtain:

$$
P\left(H_{k}, c_{i j}\right)=P_{d i j k}=\rho_{i j k} e^{-\delta_{i} \frac{r_{i}^{4}}{t_{j}}}
$$

where $\delta_{i}=-\frac{\ln \left(P_{f a}\right)}{\alpha(\cos (\theta))^{2}}$.

Since resolution cells are independent, the detection probability of the target $k$ in the direction $j$ is the sum of probabilities in the cells of this direction:

$$
P_{d j k}=\sum_{i}^{N_{r}} P_{d i j k}
$$

Finally, we determine the probability $P_{d j}$ of detecting from one to several targets in a same direction. This probability is the sum of the previous probabilities for $k$ from one to $P$. Using the Poincare formula ([26]) we obtain:

$$
\begin{aligned}
& P\left(\cup H_{i}, i \in\{1, . ., P\}\right)= \\
& \sum_{i=1}^{n} P\left(H_{i}\right)- \\
& \sum_{i, j=1, i \neq j}^{n} P\left(H_{i} \cap H_{j}\right)+ \\
& \sum_{i, j, l=1, i \neq j \neq l}^{n} P\left(H_{i} \cap H_{j} \cap H_{l}\right)-
\end{aligned}
$$

This probability should be optimized over the whole space. Unfortunately, it does not satisfy the law of diminishing returns, which is one of the assumptions of the algorithm. Moreover its expression cannot easily be used in an optimization process. A possible alternative is to make an approximation of this probability. We propose the following model:

$$
P_{d j} \simeq \exp \left(-\omega_{j} t_{j}^{-n_{j}}\right)
$$

where $\omega_{j}$ and $n_{j}$ are the modeling parameters in the direction $j$. They are determined in order to minimize the mean square error. Under this formulation, the probability has the same properties than the one given by relation (5), it is then possible to optimize it as shown in Section III, i.e. by a decompostion into an optimal number of elementary detections, and the probability of detecting from one to $P$ targets in a direction $j$ in which we spent a duration $t_{j}$ becomes:

$$
P_{d j}\left(t_{j}\right)=1-\exp \left(-\frac{t_{j}}{\tau_{j}}\right)
$$

Under this formulation, this probability satisfies the law of diminishing returns.

The assumptions of the "de Guenin" algorithm explicitly make appear the expression of the localization probability whereas it is implicitly included in the expression we have just written In order to use the algorithm, we artificially add to it:

- $p\left(c_{j}, x\left(c_{j}\right)\right)=\frac{1}{\rho_{j}}\left(1-\exp \left(-v_{j} x\left(c_{j}\right)\right)\right)$ : the local detection probability with local effort $\phi\left(c_{j}\right)$, knowing the target is in cell $c_{j}$.

- $P\left(c_{j}\right)=\rho_{j} p\left(c_{j}, x\left(c_{j}\right)\right)=1-\exp \left(-v_{j} x\left(c_{j}\right)\right):$ the detection probability in the cell $c_{j}$.

with $\rho_{j}$ the localization probability into the cell $c_{j}, x\left(c_{j}\right)$ and $v_{j}$ new notations $: x\left(c_{j}\right)=\frac{\varphi\left(c_{j}\right)}{\Phi}, v_{j}=\frac{\Phi}{\tau_{j}}$. The aim of the algorithm is then to determine $x\left(c_{j}\right), j \in\{1, . ., N\}$ such as $\sum_{j=1}^{N} x\left(c_{j}\right)=1$. 
2) Optimization: We can now carry out the optimization using the "de Guenin" algorithm. First we define the set of the cells on which the effort of research will be distributed, then we derive the expression of the elementary search effort.

Lemma 3: The cell $c_{j}$ belongs to the search space $E$ if and only if $\frac{\lambda}{\nu_{j}}<1$.

Proof: According to the "de Guenin" algorithm, a cell belongs to the search space if and only if $g(c)>\frac{\lambda}{p^{\prime}(0)}([14])$. Let us rewrite this necessary and sufficient condition using the specific notations to our problem. Since

$$
p\left(c_{j}, x\left(c_{j}\right)\right)=\frac{1}{\rho_{j}}\left(1-\exp \left(-v_{j} x\left(c_{j}\right)\right)\right)
$$

then

$$
p^{\prime}\left(c_{j}, x\left(c_{j}\right)\right)=\frac{\nu_{j}}{\rho_{j}} \exp \left(-v_{j} x\left(c_{j}\right)\right)
$$

and

$$
p^{\prime}\left(c_{j}, 0\right)=\frac{\nu_{j}}{\rho_{j}}
$$

However $g\left(c_{j}\right)=\rho_{j}$, so the two following expressions are equivalent:

$$
\left(g(c)>\frac{\lambda}{p^{\prime}(0)}\right) \Leftrightarrow\left(\frac{\lambda}{\nu_{j}}<1\right)
$$

Lemma 4: The normalized elementary search effort in a cell $c_{j} \in E_{R}$ depends on the parameter $\lambda$ and is equal to:

$$
x_{\lambda}\left(c_{j}\right)=\frac{1}{\nu_{j}} \ln \left(\frac{\nu_{j}}{\lambda}\right)
$$

Proof: The search effort is calculated by the way of the inverse of the derivative function, which is defined by:

$$
\begin{aligned}
f: \mathbb{R}^{+} & \rightarrow \mathbb{R} \\
z & \mapsto f(z)=\frac{1}{\nu_{j}} \ln \left(\frac{\nu_{j}}{z \rho_{j}}\right)
\end{aligned}
$$

According to the condition of optimality given in (33), the elementary search effort is given by the following expression:

$$
x_{\lambda}\left(c_{j}\right)=f\left(\frac{\lambda}{g\left(c_{j}\right)}\right)
$$

We have $g\left(c_{j}\right)=\rho_{j}$, so that, by combing the two previous expressions, we obtain the formulation of the elementary search effort given in the lemma 4.

The lemmas 3 and 4 enable us to establish directions in which a search effort will be spend, then we obtain the extent of this effort. The results are independent from the location probabilities $\rho_{j}$. As we said, it was implicitly included in expressions 41 , and the formulation $\rho_{j}$ was just a trick of calculation. A similar method to the one employed in the previous section could have been used. We show in [16] that they are equivalent and that they give the same results. The parameter $\lambda$ is finally interpreted like a Lagrange multiplier. However our interest for the Search Theory is double. First, it provides a theorical context, in which a great number of algorithms has been developed, and we provide it with an operational context by the use of a radar sensor, and a multitarget dimension. Note that this multitarget dimension is due for a part to the use of a radar, which is able to observe several targets in the same direction of the space simultaneously. 


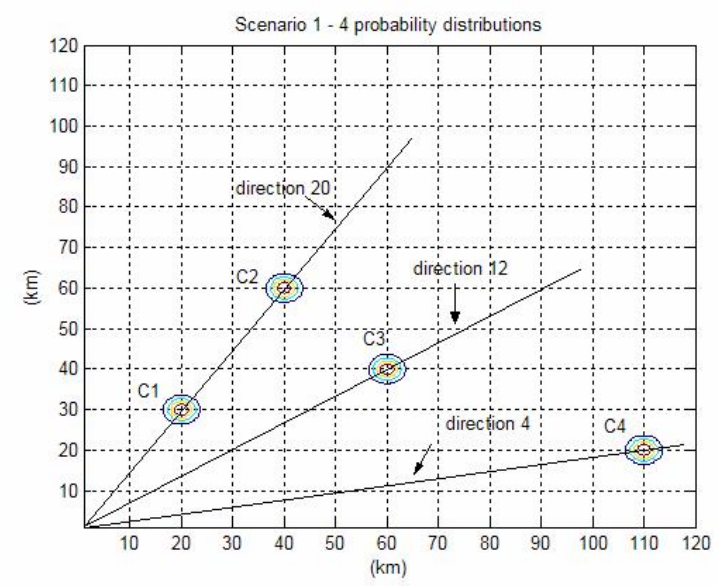

Fig. 5. Scenario 1: targets are located by distributions centers, straight lines represent the space directions

\section{ILLUSTRATIVE SIMULATIONS}

We consider four targets located in an aerial space. A prior knowledge is available for each of them, by the way of density probabilities. Figure 5 illustrates one possible scenario corresponding to the following situation:

- target 1 : the distribution is centered around the point $(20 \mathrm{~km}, 30 \mathrm{~km})$, the standard deviation on each coordinate is equal to $0.1 \mathrm{~km}$.

- target 2 : the distribution is centered around the point $(40 \mathrm{~km}, 60 \mathrm{~km})$, the standard deviation on each coordinate is equal to $0.1 \mathrm{~km}$.

- target 3 : the distribution is centered around the point $(60 \mathrm{~km}, 40 \mathrm{~km})$, the standard deviation on each coordinate is equal to $0.1 \mathrm{~km}$.

- target 4 : the distribution is centered around the point $(110 \mathrm{~km}, 20 \mathrm{~km})$, the standard deviation on each coordinate is equal to $0.1 \mathrm{~km}$.

Note that we use cartesian coordinates.

Location probabilities can be calculated from these densities by the way of an integration in the space's cells. A modeling of the detection probabilities is obtained. Modeling parameters are determined with a least square method. Table I synthetizes their values in the space directions (according to our numerics values, space is divided into forty angular directions, $N_{d}=40$ ).

\begin{tabular}{|c|c|c|c|c|c|c|c|}
\hline dir. & $1 . .3$ & 4 & $5 . .11$ & 12 & $13 . .19$ & 20 & $21 . .40$ \\
\hline$\omega_{j}$ & $+\infty$ & 56.46 & $+\infty$ & 12.45 & $+\infty$ & 1.27 & $+\infty$ \\
\hline $\mathrm{n}_{j}$ & 0 & 1 & 0 & 1 & 0 & 1.59 & 0 \\
\hline
\end{tabular}

TABLE I

Modelisation parameters in each space direction, scenarii 1

It results from this table that directions in which the couple $\left(\omega_{j}, n_{j}\right)$ is different from $(+\infty, 0)$ are potentially observable. In fact, in these directions location probabilities are not null neither negligible. It is the allocation processus which determines by the constitution of the set $E$ (lemma 3 ) which directions will actually be observed.

In Table II we can see the optimal allocation of a duration $T=30 \mathrm{~ms}$ for the detection of the targets considered in the scenario 1. The allocation is calculated by the way of results previously presented (lemmas 3 and 4). $m_{j}$ is the optimal number of elementary detections in direction $j$.

Observing Table II, we can see that only two directions are effectively considered for the temporal allocation. This is due to the allocation processus which performs a global optimization of the detection 


\begin{tabular}{|c|c|c|c|c|c|c|c|}
\hline dir. & $1 . .3$ & 4 & $5 . .11$ & 12 & $13 . .19$ & 20 & $21 . .40$ \\
\hline$\epsilon_{j}$ & 1 & 1 & 1 & 1 & 1 & 1 & 1 \\
\hline $\mathrm{t}_{j}(\mathrm{~ms})$ & 0 & 0 & 0 & 23.47 & 0 & 6.53 & 0 \\
\hline $\mathrm{m}_{j}$ & 0 & 0 & 0 & 1.31 & 0 & 2.60 & 0 \\
\hline $\mathrm{P}_{d j}$ & 0 & 0 & 0 & 0.59 & 0 & 0.97 & 0 \\
\hline
\end{tabular}

TABLE II

Optimal temporal allocation, scenarii 1, by the use of the "de Guenin" algorithm

probability. In fact, the fourth target is further from the others and from the sensor, so that too much time would be spent for its detection to the detriment of the detection of the other targets.

The coefficients $\epsilon_{j}$ which appear in table II are ponderation taps. They are all equal to one because the weighing - or priority - notion was not initially taken into account in the "de Guenin" algorithm. In the case when priority must be considered, the condition of optimality (Equation (33)) becomes $\epsilon_{j} g\left(c_{j}\right) p^{\prime}\left(\varphi\left(c_{j}\right)\right)=$ $\lambda$. The adaptation of the algorithm is possible [16]. Details are not given here but Table III shows results of optimal allocation when such factors are taken into account. In the situation we present, two targets are located in the same direction and one of them is much closer to the sensor, so that its direction is assigned a higher ponderation tap. The result is a longer observation time for this direction, and consequently a higher detection probability.

\begin{tabular}{|c|c|c|c|c|c|c|}
\hline dir. & $1 . .3$ & 4 & $5 . .11$ & 12 & $13 . .19$ & 20 \\
\hline$\epsilon_{j}$ & 0 & 0.07 & 0 & 0.18 & 0 & 0.74 \\
\hline $\mathrm{t}_{j}(\mathrm{~ms})$ & 0 & 0 & 0 & 21.08 & 0 & 8.92 \\
\hline $\mathrm{P}_{d j}$ & 0 & 0 & 0 & 0.56 & 0 & 0.99 \\
\hline
\end{tabular}

\begin{tabular}{|c|c|}
\hline dir. & $21 . .40$ \\
\hline$\epsilon_{j}$ & 0 \\
\hline $\mathrm{t}_{j}(\mathrm{~ms})$ & 0 \\
\hline $\mathrm{P}_{d j}$ & 0 \\
\hline
\end{tabular}

TABLE III

Optimal temporal allocation, scenarii 1, with consideration of ponderation taps.

\section{CONCLUSION}

The aim of this article was to present some results about resource management, in particular the allocation of a duration for the optimal detection of several targets by an ESA radar, for which the operating mode is well known. An important assumption has been formulated: the integration of the sensor in a supervision system. This led us to take into account prior knowledge about the situation to observe. During this study, several different levels of prior knowledge, including the most general and realistic one, the probabilistic case, have been successively considered. Under this prior knowledge assumption, we have proposed an analytic solution for the optimization of a criterion. This work has been carried out in the Search Theory framework. Additionally, ponderation taps have been introduced into the criterion expression, to take into account tactical considerations. Temporal allocations in order to optimize the global detection of several targets were then proposed.

All the results presented in this article are based on the modeling of the detection functions of the radar. In order to complete this work, it could be interesting to introduce another type of sensors, like infrared sensors. This type of sensor has a different operating mode. It is a passive sensor which has a mechanical scanning, for which the duration of moving the field of view cannot be neglected. Further, the concept of scheduling of the observation of the targets could be considered on top of the allocation of different priorities. Finally, we could consider the collaboration of at least two sensors, in other words the optimal temporal allocation in a multitarget multisensor context. 


\section{ACKNOWLEDGMENT}

The authors would like to thank Michel Prenat from Thales Optronics for its important contribution to this work.

The authors would like to thank Doctor Frédéric Bosché from the Computer Vision Lab, ETH Zurich, Switzerland for its important work to reread this paper.

\section{APPENDIX A}

\section{A. Signal to noise ratio}

The relation between the signal to noise ratio of the radar and the characteristics of the antenna and the sensor is given by the following expression ([19], [10]):

$$
S N R=\frac{P_{t} G_{t} G_{r} \lambda^{2} \sigma}{(4 \pi)^{3} k b F \gamma r^{4}}
$$

where:

- $P_{t}$ is the transmitted power,

- $G_{t}$ is the gain of the transmitting antenna,

- $G_{r}$ is the receive gain

- $\lambda$ is the wavelenght,

- $\sigma$ is target's radar cross section

- $k$ is the Boltzmann's constant,

- $b$ is receiver noise bandwidth,

- $F$ is receiver noise figure,

- $\gamma$ is receiver effective input noise temperature,

- $d$ is the range from the sensor to the target.

For a duration $T$, the receiver noise bandwith $b$ is equal to $\frac{1}{T}$.

$G_{o}$ is the gain of the antenna in the mechanical axis of the sensor. In a direction which forms an angle $\theta$ with the mechanical axis, the antenna's surface is multiplied by $\cos (\theta)$, so that $G_{t}$ and $G_{r}$ are equal to $G_{o} \cos (\theta)$.

Under these considerations, the expression (48) becomes:

$$
S N R=\frac{\alpha T \cos ^{2}(\theta)}{r^{4}}
$$

where:

$$
\alpha=\frac{P_{t} G_{o}^{2} \lambda^{2} \sigma}{(4 \pi)^{3} k F \gamma} .
$$

\section{B. Calibration}

$\alpha$ is a constant which depends on the characteristics of the radar and target. We suppose it is such that the signal to noise ratio of a target, located at $r=50 \mathrm{~km}$ in the mechanical axis of the sensor $(\theta=0)$ and observed during $T=5 \mathrm{~ms}$, is equal to $15 \mathrm{~dB}$. $\alpha$ is then equal to $4 \times 10^{22}$. 


\section{APPENDIX B}

During the reception chain of the radar, reception noise is added to the signal which is then transformed into composite signal. We assume that the receiver consists on an adapted filter and a detector of square signal which produces samples $\nu_{i}$ of the received power. Furthermore, we suppose that there is no postintegration, which allows us to consider only one sample $\nu$ of the received power. This random variable is normalized by the noise variance, and then compared with a threshold $S$. If there is useful signal, then there is a detection. But, if there is no useful signal, then the noise may exceed the threshold leading to a false alarm.

If there is no useful signal, the probability density of $\nu$ is equal to:

$$
p(\nu)=\exp (-\nu)
$$

The false alarm probability is the probability that the random variable $\nu$ exceeds the threshold $S$, that is:

$$
P_{f a}=\int_{S}^{+\infty} p(\nu) d \nu=\exp (-S)
$$

We call $x$ the random variable which represents the signal to noise ratio of $\nu$ and $\bar{x}$ its mean. Under the Swerling 1 modeling type assumption, the probability density of $x$ is equal to:

$$
p(x \mid \bar{x})=\frac{1}{\bar{x}} \exp \left(-\frac{x}{\bar{x}}\right)
$$

According to [17], it is possible to express the probability density of $\nu$ as:

$$
p(\nu)=\frac{1}{1+\bar{\nu}} \exp \left(\frac{-\nu}{1+\bar{x}}\right)
$$

The detection probability is the probability that the composite signal exceed the threshold $S$ :

$$
P_{d}=\int_{S}^{+\infty} p(\nu) d \nu=\exp \left(\frac{-S}{1+\bar{x}}\right)
$$

Finally, using (51), we obtain:

$$
P_{d}=\left(P_{f a}\right)^{\frac{1}{1+\bar{x}}}=\left(P_{f a}\right)^{\frac{1}{1+S N R}}
$$

\section{APPENDIX C}

The demonstration of lemma 1 is based on Karush-Kuhn-Tucker optimality conditions and consideration of a dual problem simpler to solve. It is presented in [27].

Let us use the following notations:

$$
x_{i}=\frac{t_{i}}{T} \quad \omega_{i}=\frac{T}{\tau_{r i}}
$$

With these new variables, each function $-\epsilon_{i} P_{d i}\left(t_{i}\right)$ can be written as:

$$
f_{i}\left(x_{i}\right)=\epsilon_{i}\left(\exp \left(-\omega_{i} x_{i}\right)-1\right)
$$

Let us call $\mathbf{X}$ and $f(\mathbf{X})$ the vector and the function defined by $\mathbf{X}=\left[x_{1}, x_{2}, \ldots, x_{P}\right]^{T}$ and $f(\mathbf{X})=$ $\sum_{i=1}^{P} f_{i}\left(x_{i}\right)$ respectively. The optimization problem is then transformed in a minimization problem expressed as:

$$
\mathcal{P}\left\{\begin{array}{l}
\underset{f(\mathbf{X})}{\operatorname{minimize}} \\
\text { under the constraints } \\
\left\{\begin{array}{l}
\sum_{i=1}^{P} x_{i}=1 \text { and } x_{i} \geq 0 \\
\forall i \in\{1, . ., P\}
\end{array}\right.
\end{array}\right.
$$


Constraints (19) and (20) are linear, so they are qualified. According to the Karush-Kuhn-Tucker theorem, if $\underline{\mathbf{X}}$ is the solution of the problem $\mathcal{P}$, then it exists Lagrange multipliers $\underline{\lambda} \in \mathbb{R}$ and $\left\{\underline{\mu}_{1}, \ldots, \underline{\mu}_{P}\right\} \in$ $\mathbb{R}^{P+}$ such that:

$$
\left\{\begin{array}{l}
f^{\prime}\left(\underline{x}_{i}\right)-\underline{\mu}_{i}+\underline{\lambda}=0 \\
\underline{\mu}_{i} \underline{x}_{i}=0 \forall i \in\{1, \ldots, P\}
\end{array}\right.
$$

where $f^{\prime}$ is the derivative of the function $f$ with respect to $x_{i}$.

If the suffix $i$ corresponds to a positive value of $\underline{x}_{i}$, then, according to the second line of the previous equation, $\underline{\mu}_{i}=0$. Moreover, if $\underline{x}_{i}=0$, then $\underline{\mu}_{i} \geq 0$. In both cases, the following inequality is verified:

$$
f^{\prime}\left(\underline{x}_{i}\right)+\underline{\lambda}=\underline{\mu}_{i} \geq 0
$$

that is :

$$
\left\{\begin{array}{l}
-\epsilon_{i} \omega_{i} \exp \left(-\omega_{i} x_{i}\right)+\underline{\lambda}=0 \forall i \text { such that } \underline{x}_{i}>0 \\
\epsilon_{i} \omega_{i}-\underline{\lambda} \leq 0 \forall i \text { such that } \underline{x}_{i}=0
\end{array}\right.
$$

From this equation, it results the following proposition: $\underline{x}_{i}>0$ is equivalent to $\underline{\lambda}<\epsilon_{i} \omega_{i}$. So we define the suffix set $\mathcal{I}$ :

$$
\mathcal{I}=\left\{i \in\{1, . ., P\} \mid \underline{\lambda}<\epsilon_{i} \omega_{i}\right\}
$$

and finally:

$$
\underline{x}_{i}=\left\{\begin{array}{l}
\underline{x}_{i}(\underline{\lambda})=\frac{1}{\omega_{i}} \ln \left(\frac{\epsilon_{i} \omega_{i}}{\underline{\lambda}}\right) \text { if } i \in \mathcal{I} \\
0 \text { else }
\end{array}\right.
$$

As we can see, $\underline{x}_{i}$ depends on the parameter $\underline{\lambda}$. In order to completely define $\underline{x}_{i}$, we must determine $\underline{\lambda}$. For that, we rewrite the Lagrangian function:

$$
\mathcal{L}(\mathbf{X}, \lambda)=\sum_{i=1}^{P} f_{i}\left(x_{i}\right)+\lambda\left(\sum_{i=1}^{P} x_{i}-1\right)
$$

and we define a dual problem, which has the same solutions but is easier to solve by using a classical optimization theorem. Let us call $\varphi(\lambda)$ the dual function:

$$
\varphi(\lambda)=\min _{\mathbf{X} \in \mathbb{R}^{P+}} \mathcal{L}(\mathbf{X}, \lambda)
$$

This dual function is concave since the objective function is convex. So, $(\underline{\mathbf{X}}, \underline{\lambda})$ is a saddle point of the Lagrangian function and $\lambda$ is obtained by maximizing the dual function. This dual function has a single maximum on $\mathbb{R}^{+}$because of its concavity ([25], [27]).

According to these results, the theorems presented in appendix $\mathrm{C}$, and the relation (63) between $\underline{\mathrm{X}}$ and $\lambda$, the dual function is given by the following relation:

$$
\begin{aligned}
\varphi(\lambda) & =-\sum_{i \in \mathcal{I}}\left(\epsilon_{i}-\frac{\lambda}{\omega_{i}}\right) \\
& +\lambda\left(\sum_{i \in \mathcal{I}} \frac{1}{\omega_{i}} \ln \left(\frac{\epsilon_{i} \omega_{i}}{\lambda}\right)-1\right)
\end{aligned}
$$

We introduce in this expression the function defined by relations (22):

$$
\begin{aligned}
& \varphi(\lambda)=-\sum_{i=1}^{P}\left\lfloor\left(\epsilon_{i}-\frac{\lambda}{\omega_{i}}\right)\right\rfloor^{+} \\
&+\lambda\left(\sum_{i=1}^{P} \frac{\left\lfloor\ln \left(\frac{\epsilon_{i} \omega_{i}}{\lambda}\right)\right\rfloor^{+}}{\omega_{i}}-1\right)
\end{aligned}
$$


The first derivativeof $\varphi(\lambda)$ is:

$$
\varphi^{\prime}(\lambda)=-1+\sum_{i=1}^{P} \frac{1}{\omega_{i}}\left\lfloor\ln \left(\frac{\epsilon_{i} \omega_{i}}{\lambda}\right)\right\rfloor^{+}
$$

and the single maximum is obtained by the resolution of $\varphi^{\prime}(\lambda)=0$. By the introduction of this solution $\underline{\lambda}$ in relations (63) and by the change of variables (56), we obtain the optimal temporal allocation given in the lemma. The optimal number of elementary detections is calculated by the expression (8) in which the duration $t_{i}$ is substituted to the duration $T$.

\section{REFERENCES}

[1] C. Kreucher and A. Hero, "Adaptive multi-modality sensor scheduling for detection and tracking of smart targets," Digital Signal Processing, vol. 16, pp. 546-567, September 2006.

[2] C. Kreucher, D. Blatt, A. Hero, and K. Kastella, "Non-myopic approaches to scheduling agile sensors for multitarget detection, tracking, and identification," vol. V, pp. 885-888, IEEE Conference on Acoustics, Speech, and Signal Processing (ICASSP), March 2005.

[3] Y. He and E. Chong, "Sensor scheduling for target tracking: A monte carlo sampling approach," Digital Signal Processing, vol. 16, pp. 533-545, 2006.

[4] K. Kastella, "Discrimination gain to optimize detection and classification," IEEE Transaction on Systems, Man and Cybernetics - Part A : Systems and Human, vol. 27, no. 1, pp. 112-116, 1997.

[5] C. Kreucher, K. Kastella, and A. Hero, "Sensor management using an active sensing approach," Signal Processing, vol. 85, pp. 607-624, 2005.

[6] R. Mahler, "Global optimal sensor allocation," vol. 1, pp. 167-172, Ninth National Symposium on Sensor Fusion, 1996.

[7] A. O. Hero, D. Castaãnón, D. Cochran, and K. Kastella, Foundations and Applications of Sensor Management. Springer, 2007.

[8] L. Klein, Millimeter-Wave Infrared Multisensor Design and Signal Processing. Artech House, Inc, 1997.

[9] G. W. Stimson, Introduction to Airbone Radar, Second Edition. Scitech Publishing, Inc, 1998.

[10] S. Blackman and R. Popoli, Design and Analysis of Modern Tracking Systems. Artech House, Inc, 1999.

[11] B. Koopman, "The theory of search: Part II, target detection," Operations research, vol. 4, pp. 503-531, 1956.

[12] B. Koopman, "The theory of search: Part I, kinematic bases," Operations research, vol. 4, pp. 324-346, 1956.

[13] B. Koopman, "The theory of search: part III, the optimum distribution of searching effort," Operations research, vol. 5, pp. 613-626, 1957.

[14] J. de Guenin, "Optimum distribution of effort : An extension of the koopman basic theory," Operations Research, pp. 1-7, 1961.

[15] J. P. L. Cadre and G. Souris, "Un panorama des méthodes d'optimisation de l'effort de recherche en détection," Revue Traitement du Signal, 1999.

[16] M. de Vilmorin, Contributions À la Gestion Optimale de Capteurs : Application À la Tenue de Situations Aériennes. PhD thesis, Université des Sciences et Technologies de Lille, Ecole Centrale de Lille, 2002.

[17] P. Swerling, "Probability of detecting fluctuating targets," IEEE Transaction on Information Theory, vol. 6, 1960.

[18] P. Swerling, "More on detection of fluctuating targets," IEEE Transaction on Information Theory, vol. 11, 1965.

[19] J. Darricau, Physique et Théorie Du Radar, vol. 1. Sodipe, 1973.

[20] R. Dana and D. Moriatis, "Probability of detecting a swerling 1 target on two correlated observations," IEEE Transactions on Aerospace and Electronic Systems, vol. 17, pp. 727-730, 1981.

[21] E. Duflos, M. de Vilmorin, and P. Vanheeghe, "Détermination de stratégie de gestion dynamique optimale pour un radar à balayage électronique," Revue Traitement du Signal, vol. 19, no. 2, pp. 59-73, 2002.

[22] D. Matthiesen, "Optimization of detection performance," in Proceeding of Radar 2002 Conference, IEE Conf. Publ. No.490, pp. 263269, Oct. 2002.

[23] S. Kay, Fundamentals of Statistical Signal Processing - Detection Theory, vol. 2. Prentice Hall International Editions, 1998.

[24] P. Vanheeghe, E. Duflos, P. Dumont, and V. Nimier, "Sensor management with respect to danger level of targets," (Orlando, Florida (USA)), pp. 4439-4444, 40th IEEE Conference on Decision and Control, December 2001.

[25] D. P. Bertsekas, Nonlinear programming, 2nd Edition. Athena Scientific, 2003.

[26] H. Ventsel, Théorie Des Probabilités. 1973.

[27] J. P. L. Cadre and G. Souris, "Searching tracks," IEEE, Transactions on Aerospace and Electronic Systems, vol. 36, pp. 1149-1166, 2000. 